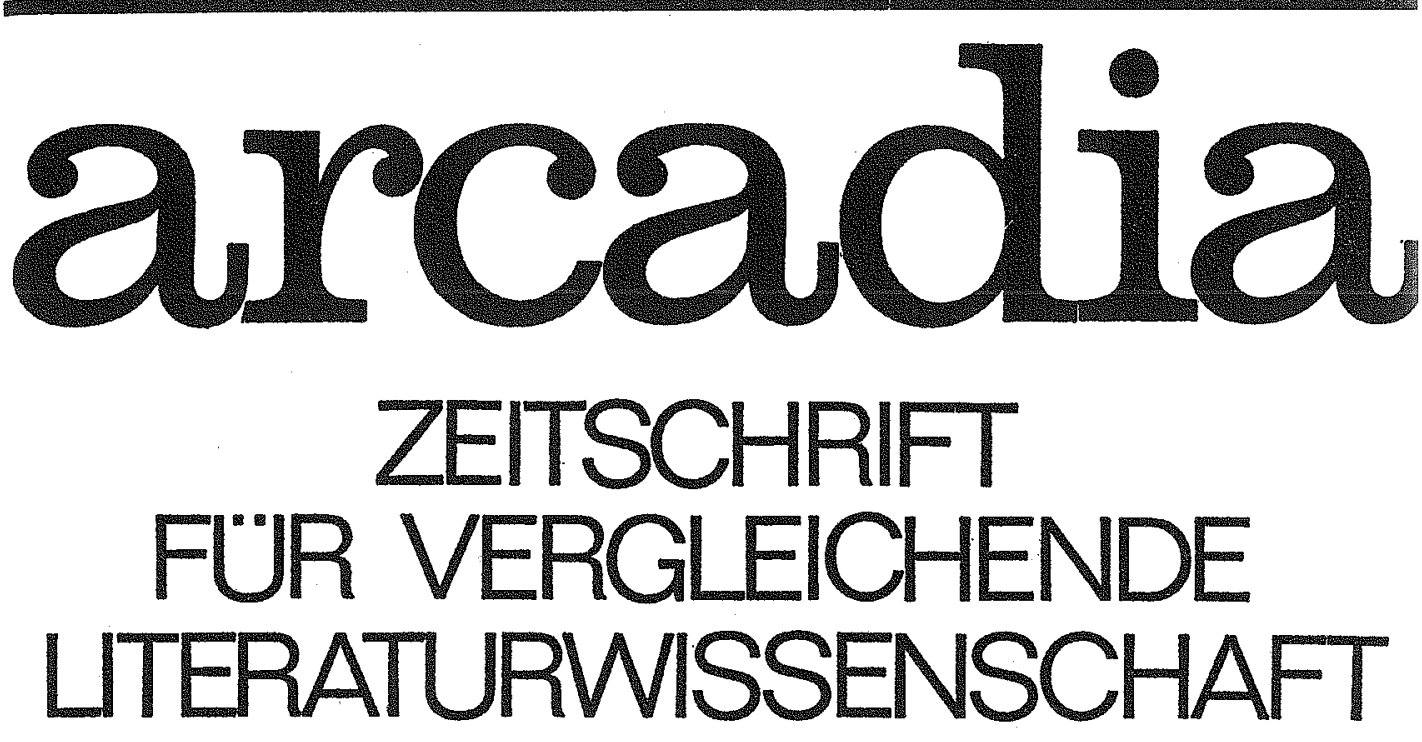

IN VERBINDUNG MIT

ROGER BAUER - WOLFGANG HOLDHEIM - ERIK LUNDING HERAUSGEGEBEN VON HORST RODIGER

BAND 141979

VERLAG WALTER DE GRUYTER - BERLIN - NEW YORK 


\section{MANFRED SCHMELING}

\section{Das ,offene Kunstwerk' in der Ubersetzung}

Zur Problematik der französischen Kafka-Rezeption

Es existiert eine sehr kurze Erzählung Franz Kafkas mit dem Titel Gibs auf! Ich möchte sie meinen Thesen zur Ubersetzung des, offenen Kunstwerkes voranstellen:

Es war sehr früh am Morgen, die Straßen rein und leer, ich ging zum Babnhof. Als ich eine Turmubr mit meiner Ubr verglich, sah ich, daß es schon viel später war, als ich geglaubt hatte, ich mußte mich sebr beeilen, der Schrecken über diese Entdeckung ließ mich im Weg unsicher werden, ich kannte mich in dieser Stadt noch nicht sebr gut aus, gliicklicherweise war ein Scbutzmann in der Näbe, ich lief zu ibm und fragte ibn atemlos nach dem Weg. Er lächelte und sagte: "Von mir willst Du den Weg erfabren?" "Ja", sagte ich, "da ich ibn selbst nicht finden kann." "Gibs auf, gibs auf", sagte er und wandte sich mit einem großen Scbwunge ab, so wie Leute, die mit ibrem Lachen allein sein wollen.

$\mathrm{Zu}$ dieser Geschichte hat Heinz Politzer einen Deutungsversuch geliefert. Kafka, so Politzer ${ }^{1}$, habe „es sich auf souveräne Art erspart, uns mitzuteilen, was der Schutzmann sich gedacht" und ob der Wegsuchende seinen Weg am Ende auch tatsächlich gefunden habe. Politzer läßr keinen Zweifel daran, daß wir es hier mit einer , offenen Form' zu tun haben. Sein Bemühen, sich den Sinn der Erzählung zu erschließen, gleicht indes mehr einer Einübung in die Methodenlehre als einer Interpretation. Alle soziologische, psychologische oder metaphysische Befragung des Textes bringt dabei als Antwort immer nur das wieder hervor, was Politzer schon vorher in die Frage investiert hatte. Mit anderen Worten: Ob das ,Ich' der Erzählung - wie Kafka im Prager Getto an "akuter Klaustrophobie" leidet oder ob es sich auf der Suche nach dem "Reich Gottes" befindet - darüber entscheidet nicht mehr der Text, sondern der Leser, sofern diesem an einer solchen Eindeutigkeits-Entscheidung überhaupt gelegen sein kann. Denn Politzer kommt zu dem Ergebnis": „Diese Parabeln sind ,Rorschachtests' der Literatur, und ihre Deutung sagt mehr über den Charakter ihrer Deuter als über das Wesen ihres Schöpfers." Was für Politzer gesicherte Erkenntnis ist - Kafka sei "nicht nur in der Wahl seiner Thematik, sondern auch in der Art seiner Darstellung [...] ein Meister der offenen Form" ${ }^{3}$ - , das wertet zum Beispiel Erwin Leibfried aus der Perspek-

1 Franz Kafka, der Künstler, Gütersloh 1965, 28.

2 Ebd. 43 .

3 Ebd. 28 . tive einer konsequenten Textimmanenz als Skandalon der modernen Literaturwissenschaf: : „Es zeugt von einer völlig mangelnden Reflexivität, Kafkas Erzählungen Vieldeutigkeit zuzusprechen; sie sind völlig eindeutig: so eindeutig, wie jeder Text vermöge seiner Texthaftigkeit zu sein in der Lage ist." Die These ist so wahr, wie die Voraussetzung, auf der sie beruht -- daß nämlich Eindeutigkeit in dem von der These implizierten Sinne aufzufassen sei. Eindeutigkeit gelingt hier um den Preis der Erkenntnis, es sei denn, daß ich meinen Erkenntnisanspruch auf die reine Textwiedergabe beschränke beziehungsweise auf eine Paraphrase in den Worten des Textes. Was aber ist dadurch gewonnen, daß ich die in der Parabel nicht beantwortete Frage nach dem Weg eben als , die nicht beantwortete Frage nach dem Weg' bezeichne? Denn das ist in letzter Instanz die Konsequenz, will man die Eindeutigkeitsrelation $\mathrm{zwischen}$ Text und Textverstehen in dem von Leibfried gemeinten Sinne aufrecht erhalten. Er bezweifelt nicht, daß der Weg auch ein Pfad sein oder zu Gott führen könne, sieht aber alle "Abschattungen“ durch ein Gemeinsames, durch "das philosophisch Weghaite“, wie er es nennt, determiniert. Nun bedeutet, Weg' freilich nicht ,Tisch', und an eine Offenheit in diesem Sinne hat Politzer gewiß nicht gedacht, sondern an einen „Horizont möglicher Bedeutungen", den Leibfried der kleinen Erzählung dann auch selber konzediert. "Vieldentigkeit" bei Polizzer heißt bei Leibfried nun "Sinn als Leerform", den es mit Bedeutung auszufüllen gelte ${ }^{5}$ : „Durch die habituelle Bedingtheit sowohl des Autors wie des Lesers werden jedoch [...] jeweils ganz bestimmte - und notwendig verschiedene - Bedeutungen konkretisiert."

Diese Auseinandersetzung zeigt die Verzerrungen, die durch den Methodenstreit auf der einen Seite und durch eine unterschiedlich gehandhabte wissenschaftliche Nomenklatur auf der anderen entstehen. Sie zeigt aber auch vor allem, daß beide Autoren eine Form der Offenheit bei Kafka anerkennen. Ein erster Blick auf die französische Obersetzung verdeutlicht die Relevanz der Fragestellung. Die Aufforderung Gibs auf! wird im Titel substantivisch mit Le renoncement, das Wörtchen Weg einmal mit chemin, einmal mit route übersetzt. Dieser an sich simple Sachverhalt zeigt nicht nur das Problem der Kafkaschen Unschärfen auf, sondern deutet bereits an, daß die Obersetzung als solche auf diese Unschärfen in viel bestimmterer Weise zu antworten gezwungen ist als die eigentliche Interpretation, die ihrem punktuellen Meinen immer durch Paraphrase Ausdruck geben kann.

Ich möchte das Problem der Offenheit bei Kafka auf folgende Gesichtspunkte eingrenzen: Erstens: Kafkas Werke zeichnen sich aus durch ein besonders hohes $\mathrm{Maß}$ an semantischer Unbestimmtheit. Auf ihr beruhen die immer neuen Interpretationsversuche. Zweitens: $E s$ muß unterschieden werden $z$ wischen einer rein semantischen Offenheit - die freilich auch bei Kafka nicht unendlich ist - und einer solchen, die die künstlerischen Darstellungsmodi betrim. Der in diesem Zusammenhang auch von Politzer verwandte Begriff der , offenen For $\mathrm{m}^{\prime}$ stiftet Verwirrung. Kafkas Werk ist, was die formallogische Struktur, also auch Dinge wie Syntax, Parabelform usw. angeht,

4 Krit. Wiss. vom Text, Stuttgart 21972, 190.

5 Ebd. 327. 
durchaus geschlossen. Und schließlich drittens: Der Eindruck der Geschlossenheit - und genan dies ist der Punkt, an dem sich die Übersetzungsqualität erweisen wird - entsteht bei Kafka aufgrund der Harmonie von Aussage und Form. Die formalen Strukturen sind in sich schon - im wahrsten Sinne des Wortes - bedeutungs-voll, denn sie sind zugeschnitten auf ganz bestimmte Inhalte.

Offenheit bei Kafka ist also nicht die Offenheit der Ausdrucksebene des Kunstwerkes. Offen erscheint dieses aufgrund des in der signifikanten Struktur immer Mit-Gemeinten. Dies ist das Gesetz der Ambiguität, durch die sich je d e ästhetische Botschaft, die Kafkasche aber in besonderer Weise, auszeichnet und die dem Rezipienten jede Sinnerfassung zu einer restriktiven Wahl macht. Kafka liefert kein bestimmtes Wirklichkeitsmodell, sondern veranlaßt den Leser, eigene Wirklichkeitsvorstellungen in das Werk hineinzuprojizieren. Die Vieldeutigkeit und Offenheit Kafkas - und ich mache mich hier zum Advocatus Diaboli der semiotischen Textwissenschaft - wäre dann weniger eine im Text selbst nachweisbare als vielmehr die Summe unterschiedlicher Rezeptionsergebnisse. In dieser Weise läßt sich wohl auch der Gedanke Umberto Ecos verstehen ${ }^{6}$, wonach "wir Leser glauben, in der Botschaft, ausgedrückt ${ }^{6} \mathrm{zu}$ finden $[\ldots]$, was wir dank ihrer Form in sie hineinlegen". Ohne diese Subjektivität, ohne diese "Aporie“, wie Eco es nennt, sind indes kein Rezeptionsvorgang und keine Kommunikation denkbar; daß man sich der Aporie bewußt ist, das dürfte hier der wesentlichere Gesichtspunkt sein. Diese Situation ist übertragbar auf die Ubersetzung. Denn - und das ist ein hinreichend bekannter Sachverhalt, den ich hier nicht theoretisch zu explizieren brauche - die Ubersetzer sind immer auch Ausleger und Interpreten, wobei die Rezeption einerseits schon vor dem eigentlichen Übersetzungsakt beginnt, andererseits mit diesem noch keineswegs abgeschlossen ist. -

Als Vermittler Kafkas in Frankreich treten zunächst die Surrealisten und ihnen nahestehende Autoren auf. Sie leiten die erste Phase französischer KafkaRezeption ein, die sich, dann vor allem durch die ontologische Kritik Camus' und Sartres geprägt, bis in die fünfziger Jahre hinein fortsetzt. Im künstlerischen Bereich ist dieser Rezeptionsart insbesondere Gide verpflichtet, der, auf der Grundlage der Ubersetzung von Alexandre Vialatte, den Prozeß-Roman in ein Bühnenstück umgeschrieben hat. Josef $\mathrm{K}$. wird hier in die Rolle des existentialistischen Helden gezwängt. Die Ereignisse des Krieges und die Nachkriegszeit bewirken indes auch schon eine Horizonterweiterung in Richtung auf soziologisch-gesellschaflich orientierte Verstehensweisen. Erst mit einiger Verspätung und nicht ohne Mitwirkung der Nouvelle Critique und des ihr verbundenen Nouveau Roman wird schwerpunktmäßig dem artistischen Können Kafkas, seiner Sprache und der darin sich äußernden Kunstauffassung Aufmerksamkeit geschenkt. Hierfür stehen Namen wie Marthe Robert, Roland Barthes und Robbe-Grillet. Den vorläufigen Schlußpunkt setzen die Philosophen Gilles Deleuze und Félix Guattari, die den besonderen Idiolekt Kafkas als Politikum verstehen und seine Werke in eine Minderheitenliteratur einreihen, in eine, littérature mineure ${ }^{c}$ mit revolutionärem Impetus.

\footnotetext{
- Einfübrung in die Semiotik, München 1972, 155.
}

Was sich hier also andeutet, ist die Vielschichtigkeit der durch Rezeptionsgemeinschaften vollzogenen Konkretisationen, die, wie gesagt, mit dem Surrealismus beginnen. Offenbar stand die Obertragung der Verwandlung (durch Alexandre Vialatte in der Nouvelle Revue Française, 1928) und des Urteils (durch Pierre Klossowski und Pierre Leyris in der Zeitschrift Bifur, 1930) in direktem Zusammenhang mit einer Einschätzung Kafkas als eines der surrealistischen Asthetik zumindest nahestehenden Autors. Die Æ̈hnlichkeiten $z$ wischen der surrealistischen Poetik und Kafka sind freilich, soweit sie Die Verwandlung und Das Urteil betreffen, vorwiegend thematischer Natur. Sie gruppieren sich um die Phänomene Traum, Erotik, Humor, Wahnsinn, Selbstmord und Revolte, wobei der gemeinsame Strang zur Freudschen Psychoanalyse eine bestimmende Rolle spielt. Weniger bewußt war den Kommentatoren und Übersetzern, daß das Phänomen der Dissoziation des Ichs auch eine gesellschaftspolitische Problemseite und als solche ihren Stellenwert in der Kunst Kafkas besaß37. Die Konzentration auf eine eher spektakuläre Thematik hat dann aber vor allem eine Vernachlässigung der sprachlichen Seite zur Folge - und das wirkt sich direkt auf die Übersetzungen aus.

Der fatale Inhaltswert dieser Sprache, also besonders ihre Vieldeutigkeit und Offenheit, lassen Kafkas Wirkung aufgrund von Ubersetzungen zumindest problematisch erscheinen. Ich möchte die Problematik zunächst an einem Beispiel aus Das Urteil kurz illustrieren. Es geht um den letzten Satz der Erzählung, die mit dem Selbstmord Georg Bendemanns schließt ${ }^{8}:$ In diesem Augenblick ging über die Brücke ein geradezu unendlicher Verkebr. In der Ubersetzung von Pierre Klossowski und Pierre Leyris lese ich folgendes ${ }^{9}: A$ ce moment commence sur le pont une circulation interminable. Michel Carrouges variiert in seinem Buch über $\mathrm{Kafka}^{10}:$ A ce moment, il $y$ avait sur le pont une circulation littéralement folle.

Im deutschen Satz ist die Aktionsart durativ mit leicht iterativer Tendenz. Sie relativiert einerseits den punktuellen Einstieg (In diesem Augenblick) und bedeutet andererseits Affirmation der ,Unendlichkeit ${ }^{c}$ des Geschehens. Die erste französische Variante verändert die Aktionsart durch das inchoative commence, die zweite beschränkt sich auf eine rein durative Form (il y avait), versucht aber diesen Unterschied in der Aktionsart des Verbs mit Hilfe einer Umstilisierung - der unendliche Verkehr wird zum "wahnsinnigen" Ver-

7 Das hing sicherlich z.T. auch mit dem Mangel an hermeneutischer Distanz zum Interpretandum zusammen. Allerdings bezieht sich dieser Mangel weniger auf das interliterarische als auf das historische Gefälle, denn ein Blick auf deutsche Rezensionen zeigt ganz ähnliche, mitunter noch eindeutigere Sinngebungen. Am Proze $\beta$ beispielsweise interessiert einen Interpreten - es handelt sich um einen Kommentar Peter Panters [d. h. Kurt Tucholskys] aus der Weltbïhne I (1926) 384 f. die Szene, wo ,der lederschwarze Prügler, aus einer Masochisten-Photografie geschnitten, die Rute schwingt..."; es ist die Rede vom "Tagtraum", vom "vernünftigen Irren“, von der "Angst, die der Materie in alle Poren brüllt" liches mehr.

8 Die deutschsprachigen Zitate aus dem Werk Kafkas beziehen sich auf folgende Ausg.: Franz Kafka: Ges.W. I-VII, hg. v. Max Brod, Frankfurt 1976. P. = Der Prozeß, Sch. $=$ Das Scbloß.

- In: Bifur, Juli 1930.

10 Franz Kaflea, Paris 1948, 28. 
kehr - mehr als gutzumachen. Das einschränkende geradezu bleibt in der ersten Variante unübersetzt. So viel zur stilgrammatischen Deskription der Obersetzungsvarianz, die für die Interpretation des Wörtchens Verkebr durchaus bedeutsam ist. Daß es sich dabei um ein Schlüsselwort Kafkas handelt, fällt zunächst nur demjenigen auf, der die anderen Werke Kafkas, den biographischen Hintergrund sowie bestimmte Rezeptionsergebnisse kennt. Natürlich ist an der Ubersetzung von Verkebr durch circulation philologisch nichts auszusetzen. Nur hat eben ,Verkehr' im Deutschen noch andere Konnotationen, über die sich ein Autor mit dem Sprachbewußtsein Kafkas im klaren war. Den eigentlichen Anstoß gibt indes der Dichter in einem Gespräch mit Brod. Er soll diesem anläßlich des Urteils gesagt haben ${ }^{11}$ : Weißt, du, was der Schlußsatz bedeutet? Ich habe dabei an eine starke Ejakulation gedacht. Dieser psychoanalytische Hinweis wird in einer Tagebuchstelle, auf die ebenfalls Brod aufmerksam macht, mit dem Akt der künstlerischen Produktion verknüpf ${ }^{12}$ : [...] die Geschichte ist wie eine regelrechte Geburt mit Schmutz und Scbleim bedeckt aus mir herausgekommen, und nur ich babe die Hand, die bis zum Körper dringen kann.

Ich kann es mir ersparen, dem Wörtchen Verkebr, das bei Kafka oft genug belegt ist, häufig auch als Parteienverkebr, in seinen eventuell zweideutigen Bezügen nachzuspüren ${ }^{19}$. Dies hat unter anderem Politzer - anläßlich desselben Paradigmas - mit gebührender Vorsicht getan ${ }^{14}$. Worauf es hier ankommt, ist zu zeigen, wie die konnotative Disponibilität der Kunst Kafkas in der Ubersetzung, in diesem Falle zwangsläufig, eingeschränkt wird. Daraus resultiert ein zweites: Carrouges läßt der Ubersetzung des betreffenden Satzes, von der nicht ganz klar ist, ob sie seine eigene oder eine kolportierte ist, eine Interpretation folgen, die von der poetischen Wirkung dieses Satzes ausgeht ${ }^{15}$ : "Quel est l'être sensible à la poésie qui pourrait ne pas ressentir l'éclat de cette ultime phrase, retentissante comme un coup de gong." Gleichzeitig aber ist er sich der Kafkaschen Polyvalenz durchaus bewußt ${ }^{16}:$, [...] il utilise en général des phrases aussi nues et simples que celles des vrais classiques, mais il les valorise à un degré inusuel par le sens caché qu'il leur donne, par la vibration poétique extraordinaire qu'il en tire." Unser Vergleich hat indes verdeutlicht, daß - abgesehen von den sonst festgestellten syntaktisch-semantischen Unterschieden - zumindest e in ,sens cachée sich nach der Übersetzung des Satzes nicht mehr ohne weiteres rekonstruieren läßt, die zielsprachliche Fassung also hinter dem Original zurückbleibt. Anders ausgedrückt: Die Übertragungen von Carrouges und Klossowski/Leyris schließen bestimmte, etwa Politzers Uber-

11 Max Brod: Franz Kafka - Eine Biogr., Frankfurt/M. 1962, 158.

12 Ebd

1s Vgl. zum obigen Problem die ebenso bedrückende wie erotische Atmosphäre im dritten Kapitel des Prozesses, das mit dem Zusammentreffen zwischen Josef K. und dem M⿱丷己心dchen im Dachboden endet. Vor diesem Hintergrund stimmen die folgenden Sätze zumindest nachdenklich $(P .63)$ : „Hier können wir nicht bleiben, bier stören wir den Verkehr" $-K$. fragte mit den Blicken, welchen Verkebr er denn bier störe.

14 AaO. [Anm. 1] 97

15 Carrouges [Anm. 10] 28.

15 Ebd.29f. legungen zum Verkehrsproblem in den Werken Kafkas aus und kompromittieren, was den punktuellen Aussagebezug betriff, den Selbstkommentar des Dichters. Um so kurioser erscheint daher, daß Carrouges, in Kenntnis der Information Kafkas bzw. Brods, dann doch die Komponente des „arrière-plan érotique $^{\text {"17 }}$ - freilich an anderer Stelle - wieder ins Spiel bringt, ohne simultan die Obersetzung zu problematisieren.

Dieses - zugegebenermaßen etwas frivole - Beispiel hat insofern einen ernsten Hintergrund, als es die Möglichkeiten und Grenzen der Kafka-Übersetzungen und der damit verbundenen Rezeption punktuell aufzeigt. Freilich ist die Problematik der Kafka-Rezeption in Frankreich sehr viel komplexer. Einen besonderen Raum nehmen dabei die drei Romane ein, die von Claude David erst kürzlich (1976) in der Pléiade neu ediert ${ }^{18}$, aber - und die Konsequenzen dieser Entscheidung sind noch nicht abzusehen - nicht neu übersetzt wurden. Daß die über vierzig Jahre alten französischen Fassungen - Vialattes Ubersetzung des Prozesses stammt aus dem Jahre 1933 - wahrscheinlich auch die nächsten fünfzig Jahre überleben werden, ist die Folge eines juristisch abgesegneten Einspruchs der Vialatte-Erben, die das Andenken des Übersetzers auf ihre Weise respektieren. Aus diesen Gründen mußte sich David auf mehr oder weniger punktuelle Korrekturen beschränken, die der Normalleser kaum zur Kenntnis nehmen dürfte. Aber auch auf dieser Basis ist ein grundsätzliches hermeneutisches Problem erkennbar. Der Einfluß der zwischenzeitlich fortgeschrittenen wissenschafllichen Rezeption läßt sich bei David auf Schritt und Tritt belegen. Ein solches Verfahren, in dem eine in der Sache berechtigte Ubersetzungskorrektur ihre angebotenen Varianten unter anderem durch Zitate aus der kritischen Literatur und durch eigene Interpretationen - David hat als Kafka-Interpret internationalen Ruf - beglaubigt, mag auf den ersten Blick etwas befremden. Aber das Gesetz der Historizität wirkt sich hier durchaus auch positiv aus. Sowreit es sich um im Laufe der Geschichte angesammelte Meinungen, Interpretationen, auch um objektive, zum Beispiel biographische Erkenntnisse handelt, wird der Prozeß der Fremdbestimmung in den Werken Kafkas explizit und kann vom Leser nachgeprïft werden. Die ersten Ubersetzer konnten, unter anderem wegen des Rezeptionsvakuums im Dritten Reich, auf derartigen Voraussetzungen nicht aufbauen. Dies allein ist freilich noch keine Erklärung fü: die Öbersetzungsvarianz, wie die folgenden Überlegungen vielleicht deutlich machen werden.

$\mathrm{Zu}$ den sprachlichen Besonderheiten in den Werken Kafkas zählen auch die ersten und letzten Sätze seiner Romane und Erzählungen. Insbesondere gilt dies für den Prozeß, dessen erster Satz, wie man weiß, die Tonlage kommender Geschehnisse bestimmt. Seine Schlüsselrolle in der Textsemantik ist in etwa vergleichbar mit der Rolle, die die Türhüter-Episode im hermeneutischen Selbstverständnis des Romans spielt (P. 7): Jemand mußte $K$. verleumdet haben, denn obne daß er etreas Böses getan bätte, wurde er eines Morgens

17 Ebd. 103.

18 Franz Kafka: Evures compl. I - Trad. par Alexandre Vialatte - Ed. présentée et annotée par Claude David, Paris 1976. (Die französ. Fassung der Romane wird nach dieser Ausg. zitiert.) 
verbaftet. Der hypothetisch-affirmative Stil dieses Satzes wird von Kafka konsequent bis zum letzten Satz, dem durch Mord erzwungenen Ende des Josef K., durchgehalten (P. 193): "Wie ein Hund!" sagte er, es war, als sollte die Scham ibn überleben. Diese Symmetrie signalisiert eine, zunächst nur textmorphologisch und stilistisch $z u$ verstehende, äußerlich u. a. durch das modale mußte bzw. sollte ausgewiesene Geschlossenheit des Romans. Der strukturelle Gleichlauf, ebenso wie die damit zusammenhängende Technik des medialen Erzählens, die den Leser zum unmittelbaren Vertrauten der Vermutungen und Meinungen Josef K.s macht, verdeutlichen noch einmal, daß von einer, offenen Form' in der Darstellung zumindest im Proze $\beta$ nicht gesprochen werden kann. In der französischen Ubersetzung Vialattes beginnt der Roman so (259): On avait sûrement calomnié Joseph $K$., car, sans avoir rien fait de mal, il fut arrêté un matin. Die für Kafka so typische Unentschlossenheit zwischen hypothetischen und affirmativen Wendungen verschiebt sich in der Ubersetzung des Satzes zugunsten der Affirmation (indikativischer Einstieg, sĥrement, Infinitiv - nur am Rande erwähnt sei hier die wenig Kafkasche Kakophonie von [...] calomnié Josef $K$., car [...]). Wo also der deutschsprachige Satz unter Verwendung perspektivischer Ambiguität (u. a. ausgedrückt durch die Modalverben und den Konjunktiv) Unbestimmtheit diktiert, spiegelt die Ubersetzung, indem sie die Aussage genau macht, gesicherte Information vor. Die festgestellte Abweichung erlaubt noch keine allgemeinen Schlïsse zur Ubersetzungsstrategie. Sicher ist, daß wir es in diesem Fall nicht nur mit einer Insuffizienz der Zielsprache $z u$ tun haben, die den Kafkaschen Stil nicht wiederzugeben vermöchte. David stellt in seiner Variante das Gleichgewicht zwischen Original und französischer Fassung in etwa wieder her (Quelqu'un avait dî calomnier Josef K. [...]).

Im siebenten Kapitel des Prozesses erreicht der kafkaeske Vermutungsstil einen echten Höhepunkt. Die perspektivische Ambiguität wird nun in durchaus programmatischer Absicht betrieben, indikativische und konjunktivische Wendungen sind destruktiv aufeinander bezogen. So wird das von der Aspektfigur Josef K. kolportierte Gespräch mit Advokat Huld, nur scheinbar willkürlich, bald im Konjunktiv, bald im Indikativ wiedergegeben. Einmal erscheint das Wissen über den Proze $\beta$ als ein von Josef $K$. erschlossenes bzw. vermitteltes - und wie der reine Irrealis besitzt der Zitat-Konjunktiv gegenüber objektiven Sachverhalten eine einschränkende Funktion -; dann wieder wird beim Leser, augenblickhaft, der Eindruck einer objektiven Wahrheit vorgetäuscht: Hier mache sich eben der Nachteil einer Gerichtsorganisation geltend (P. 102), Den Beamten feblt der Zusammenhang der Bevölkerung (P. 102), Die Rangordnung und Steigerung sei unendlich und selbst für den Eingeweibten nicht absebbar (P. 103). Nun die Ubersetzung: C'était là qu'spparaissait justement le défaut d'une organisation judiciaire (365), Les fonctionnaires manquaient de contact avec la société (366), La biérarchie de la justice comprenait des degrés infinis au milieu desquels les initiés eux-mêmes avaient peine à se trouver (366).

Die französische Ubersetzung kann diese Ambiguität des Erzählens, die den unsicheren Standort Josef K.s in der Prozeßwelt spiegelt, nur partiell ausdrücken. Kafka hingegen hat diese Kongruenz zwischen der Ausdrucksebene und der Inhaltsebene bewußt forciert. Dem „eigentümliche[n] Schweben der Darstellungsweise [...] zwischen objektiver Vorgangsschilderung und subjektiver Perspektive" (Beda Allemann ${ }^{19}$ ) entsprechen inhaltlich Außerungen wie: Einzuseben versuchen, daß dieser große Gerichtsorganismus gewissermaßen ewig in der Schwebe bleibt (P. 104); Dann scheint einem allerdings nichts mebr sicher (P. 105); dessen dürfe K. sicher sein (P. 106), oder Immer gab es Fortschritte, niemals aber konnte die Art dieser Fortscbritte mitgeteilt werden (P. 107). Die Abweichung ist weitgehend durch die Ausdrucksmöglichkeiten des Französischen bedingt, das hier den deutschen Zitat-Konjunktiv durch den Indikativ des Präteritums wiedergeben muß; sie bewirkt eine deutliche Veränderung in der syntaktisch-semantischen Struktur des Romans. Die Form-Inhalt-Kongruenz, die, wie ich zu Beginn sagte, den Eindrudk von Geschlossenheit hervorruft, wird von der Ubersetzung gerade nicht eingelöst.

Eine weitere Beobachtung läßt sich anschließen. Während im deutschen Text der Konjunktiv signalisiert, daß es sich jeweils um eine durch die Aspektfigur Josef $K$. gefilterte Aussage des Advokaten handeln muß, läuft das Subjekt der Aussage im französischen Text wegen der Länge des indirekten Berichtes Gefahr, beim Leser in Vergessenheit zu geraten. Vialatte war sich dieser Gefahr bewußt und versucht, dem Leser eine Orientierungshilfe dadurch zu geben, daß er in Abweichung vom Original mehrfach Formeln einfügt wie ajoutait l'avocat (366) oder disait le docteur Huld (367). Diese Amplifikationen machen die im deutschen Text nur verdeckt vorhandene metasprachliche Ebene explizit. Daraus folgt notwendig eine Disziplinierung und Objektivierung textinterner kommunikativer Strukturen, die sich freilich auch auf die Kommunikation $z$ wischen Text und Leser auswirkt ${ }^{20}$. Ambiguität und Mißverständlichkeit in den Kommunikationsabläufen zwischen $K$. und seiner Umwelt - und das gilt auch für den Schloß-Roman - haben indes bei Kafka gerade die Funktion, Verstehensweisen oder Sehweisen zu problematisieren. Ein Beispiel, das die Adverbien des Meinens betriff - und damit schließe ich diesen Punkt ab mag die Ubersetzungsproblematik in diesem Zusammenhang nochmals verdeutlichen (Sch. 238): Wobl aber tue ich ibm vielleicht Unrecht, das ist freilich möglich. Vialatte verkürzt (745): Mais que je sois injuste, c'est possible.

19 Kafka - Der Prozeß, in: Der dt. Roman II, hg. v. B. v. Wiese, Diisseldorf 1965 , 234-290, hier: 240 .

20 Für dieses Phänomen lassen sich zahlreiche weitere Beispiele anführen. So heißt es im franz. Text anläßlich desselben Gesprächs mit Huld (368): Il fallait éviter à tout prix de se faire remarquer, rester tranquille même si on y éprouvait la plus grande répugnance, tâcher de comprendre que [...]. Im Original steht (P. 104): Nur keine Aufmerksamkeit erregen! Sich rubig verbalten, selbst wenn es einem noch so sebr gegen den Sinn gebt! Einzuseben versuchen, daß [...]. Nicht nur werden bei Vialatte die drei elliptischen Konstruktionen in einer einzigen Periode aufgelöst, sondern die Erzähldimension als solche ist von der Variante $I l$ fallait [...] que betroffen. Im deutschen Text haben wir es mit Sequenzen zu tun, die - scheinbar - spontan artikuliert sind und in ihrer Erlebnisform, $j$ a bis in die Wortfolge und Wortwahl hinein an die subjektivste aller literarischen Ausdrucksweisen, an das Tagebuch erinnern, an Sätze wie: Nur nicht überscbätzen, was ich gescbrieben babe [...]. Eingeseben, daß ich die Zeit seit Awgust durchaus nicht geschrieben babe [...], Eingeseben, caßs ich die Zeit seit August durchaus nich h. v. E. Heller u. J. Beng, München 1969; hier: 124 und 142). 
Die Adverb-Akkumulation ist hier nicht nur Ausdruck für den hypothetisch-affirmativen Stil. Sie ist noch für eine weitere wichtige Erscheinung in der Sprache Kafkas kennzeichnend: die Redundanz. Wie das Beispiel eben schon andeutete, benutzt er die Redundanz gerade nicht zur "Sicherung der Nachrichtenübermittlung" - etwa im Sinne des bekannten Beispiels aus der Minna von Barnhelm ${ }^{21}$ : ...aber allein, ganz allein, insgebeim, unter vier Augen. Vielmehr besitzen die redundanten Strukturen vor allem im Proze $\beta$ zunächst einmal eine - freilich vom Dichter beabsichtigte - negative Funktion: Sie verhindern Eindeutigkeit. Es gilt außerdem zu unterscheiden $z$ wischen einer Redundanz auf der, signifiant'-Ebene und einer Redundanz auf der ,signifiée-Ebene. Bei Kafka manifestiert sie sich vor allem auf der ,signifiant'Ebene, und die mehrfache Wiederholung eines bestimmten Lexems bedeutet nicht notwendig die Wiederholung desselben ,signifié. Ich möchte diesen etwas kompliziert ausschauenden Sachverhalt und seine Rezeption in der Ubersetzung folgendermaßen verdeutlichen: Eine Frequenz-Analyse der Türhüter-Episode im Prozeß ergibt im Original zwölfmal die Verwendung des Wörtchens Meinung; in der Ubersetzung Vialattes hingegen finde ich folgende Varianten: dreimal thèse, zweimal gloses, einmal commentateur (personal), einmal Voilà qui va loin (idiomatisch umschrieben), zweimal avis, zweimal opinion und einmal sentiment. Die Veränderung resultiert aus einer unterschiedlichen Bewertung der Redundanz. Letztere wird bei Vialatte auf der signifiant-Ebene weitgehend aufgehoben. Freilich ist damit auch eine Manipulation der inhaltlichen Aussage verbunden. Die im Wort Meinung mitschwingenden Bedeutungen, die der Leser erschließen muß - und darin äußert sich die Liberalität der Werke Kafkas —, diese nur potentiell vorhandenen Bedeutungen werden zum Teil in der Obersetzung explizit, das heißt durch bestimmte Signifikanten realisiert. Vialatte tut also genau das, was durch das Lessing-Beispiel ausgedrückt wird: Er versucht, die Information genau zu machen. Das führt jedoch letztlich zum Informationsverlust für den Empfänger der poetischen Botschaft ${ }^{22}$. Die mit diesen Varianten verbundenen semantischen Einkreisungen Vialattes widersprechen der in der Auslegung des Gleichnisses zum Ausdruck kommenden Strategie, die hermeneutische Situation offen zu gestalten. Durch die Form seiner Darstellung, durch die semantische Indifferenz der Begriffe - und sie wird mit Hilfe der Redundanz ja noch verstärkt - produziert Kafka einen ähnlichen Bedeutungsfreiraum für den Leser wie der Geistliche für Josef $K$. Anders ausgedrückt: Kafka hat in vollem Bewußtsein des hermeneutischen Problems solche gestalterischen Mittel, also Redundanz, Unbestimmtheit, Gleichnisform usw, eingesetzt, die den Akt des Verstehens in seinem Text erschweren. Begriffe wie glose oder glossateur, die eindeutige Konnotationen erwecken, sind einer solchen Strategie nicht hilfreich. Diese Erkenntnisse lassen

21 Vgl. Theodor Lewandowski: Linguistisches Wb. III, Heidelberg ${ }^{2} 1976,568$.

22 Ahnlich verfährt der Obersetzer mit dem wichtigen Wort täzschen bzw. Täuschung, das wiedergegeben wird durch méprendre, erreur oder tromper; für Türbüter schließlich steht sentinelle, gardien und portier. sich auf zahlreiche andere Beispiele übertragen, in denen die Redundanz reduziert und Unbestimmtheit in Bestimmtheit verkehrt wird ${ }^{23}$.

Solche Beobachtungen bestätigen das Selbstverständnis des Übersetzers als Interpreten. Kann man nun sagen, daß sich hinter den Interpretationen - und ich beziehe mich zunächst auf die Übersetzungen der Romane Der Prozeß und Das Scbloß - ein bestimmtes System verbirgt, kann von einem spezifischen Interpretationsstandpunkt, somit von tendenziöser Ubersetzung die Rede sein? Erste Hinweise hierfür gibt das mit den Romanen veröffentlichte kritische Akzessorium. Die in meinem Besitz befindliche Ausgabe des Prozesses ${ }^{24}$ enthält (neben der Ubersetzung) einen etwa vierzigseitigen Kommentarteil, der unter anderem aus einer Préface des Philosophen Bernard Groethuysen, einer Introduction von Vialatte und dem bekannten Postskriptum von Max Brod besteht. An Brods eindeutige Stellungnahmen sei in diesem Zusammenhang nur erinnert; sie haben mitgewirkt an dem, was ich den ,metaphysischen Geist' der Ubersetzung nennen möchte. Zentraler Gesichtspunkt in der Einführung Vialattes - und die theologische Autorität von Max Brod wird ausdrücklich hervorgehoben - ist das Motiv der Erbsünde („,coupable-néc c $^{\prime}$ und die Suche nach Gott („la quête de Dieu") ${ }^{25}$. In dieser Einführung ist die Übersetzerintention deutlich formuliert, und jeder Literaturwissenschaftler, der sich mit Obersetzungen befaßt, ist für derartige Materialien dankbar. Denn ein bestimmtes Konzept aus der Ubersetzung selbst herauszulesen, wird mitunter zu einer äußerst schwierigen Aufgabe. Die hermeneutische Úbersetzungswissenschaft hat bei der Suche nach Kriterien und Methoden zur Feststellung dieses Phänomens m. E. noch zu wenig differenziert. Erwähnung finden vor allem die beiden Pole, auf denen der Ubersetzer sein Konzept alternativ oder zwischen beiden vermittelnd aufbauen kann. Einesteils geht es darum, die Eigenarten des Originals, das in einen bestimmten historischen Prozeß eingegliedert ist, $\mathrm{zu}$ erfassen und mit den Ausdrucksmöglichkeiten der Zielsprache neu zu konkretisieren. Das historische Treueideal betrifft aber nur die eine Seite des Ubersetzungsprozesses, der sich immer auch den Bedingungen der aufnehmenden Kultur anzupassen hat. Dieser

${ }^{23}$ Im 5. Kap. des Prozesses, überschrieben mit Der Prügler, sind die Worte Prïgler bzw. priigeln achtzehn Mal belegt. Vialatte liefert die tollsten Varianten von fonetter ibber fustigeur bis hin zur Kapitelüberschrift Le bourreau. Angesichts dieser fonetter uber fustigenr bis hin zur Kapitelüberschrift Le bourreau. Angesichts dieser Anderungen fühlt man sich an die Worte des Geistlichen erinnert, der zu Josef $K$. sagt ( $P .183$ f.): Du bast nicht genug Achtung vor der Scbrift und veründerst die
Geschichte. Geschichte.

24 Franz Kafka: Le Procès. - Nouvelle éd., Paris 1957.

Die philosophische Textbezogenheit des kritischen Apparates der von David bearbeiteten Kafka-Ausgabe spiegelt eine nicht ganz so eindeutige, aber trotzdem tendenziöse Auslegung wider. Die Erkenntnisse über den $S c h l o ß$-Roman beispielsweise lassen auf eine zwar undogmatische, allgemeinere Verstehensweise schließen, doch ist die religiöse Komponente dabei dominant. Kafka gilt als „un esprit religieux parmis les plus profonds de notre temps" (1138), eine Einschätzung, die im Verständnis des Textes als Gottsuche ("Ce Dieu que cherche $K . “[1133]$ ) grinndet. Punktuell zeigt sich auch bei David eine Tendenz zur Entweltlichung von Gestalten des Romans, etwa wenn der Bote Barnabas als "Engelsbote" (l'ange porteur de bonnes nouvelles" [1159]) apostrophiert wird. Davids pro-mystisches Interesse an Kafka äußert sich $u$. a. in folgendem Urteil (1138): "Comme les mystiques, il joue dangereusement avec le vide; comme eux, il s'écarte des dogmes et frôle constamment le scandale, comme eux aussi, il se prête à tous les malentendus et tous les contresens." 
zweite funktionale Aspekt wird übrigens besonders von der marxistischen Literaturkritik hervorgehoben, die freilich mit Anpassung an die veränderten Verhältnisse eine ganz spezifische ,Parteilichkeit des Übersetzers meint ${ }^{26}$. Angesichts der Frage nach der Rolle der Übersetzung in den internationalen literarischen Beziehungen wäre also auch zu überprüfen, ob überhaupt und nach welchen Regeln von Ubersetzungsvarianz auf bestimmte Konzeptionen geschlossen werden kann. So viel scheint sicher: Die Übersetzerintention wird desto deutlicher, je konsequenter sie an einem System partizipiert; dieses muß nicht politisch-gesellschanlich verankert sein, sondern kann zum Beispiel - so bei Vialatte - einer metaphysischen oder religiösen Ordnung angehören. Hier wie dort enthält sie eine stark ideologische Komponente.

Eine Ubersetzung sollte man dort nach möglichen Vorurteilsstrukturen befragen, wo die größte hermeneutische Belastung $\mathrm{zu}$ vermuten ist. Eine echte Bedeutungskonzentration begegnet uns in dem Begriff der,Schuld', einem der wesentlichsten Schlüsselwörter der Romane Kafkas. Man kann absehen, was es für den Leser bedeutet, wenn in der französischen Fassung Schuld teils aufgrund des Kontextes, teils willkürlich mit culpabilité, délit, faute oder crime wiedergegeben wird. Diese Ambivalenz zwischen der konkret bestimmbaren und einer allgemeinen Schuld wird im deutschen Text begrifflich nicht akualisiert. Allein die Tatsache, daß die K.s immer wieder nach Erklärungen und Ursachen für ihre aktuelle, desolate Lage suchen, läßt auf ihr Verständnis von Schuld als rechtliches oder moralisches Vergehen (délit oder crime) schließen. Daß Vialatte hier einen echten Konflikt auszutragen hatte, zeigt das folgende Beispiel. Die Mehrdeutigkeit des, Schuld'-Begriffes wird offenbar erfaßt, kann aber nicht ohne Verschiebung in der semantischen Struktur in die französische Sprache umgesetzt werden (P.109): Vor allem war es [..] notwendig, jeden Gedanken an eine möglicbe Scbuld von vornberein abzulebnen. Es gab keine Scbuld. Ubersetzung (372): Il était surtout nécessaire [...] d'éliminer à priori toute idée de culpabilité. Il n'y avait pas de délit. Die Tatsache, daß zwei semantisch divergierende Ausdrücke (culpabilité, délit) verwandt werden, entsprich technisch gesehen dem bekannten Sachverhalt: Während wir es im Original mit einem Fall von polyfunktionaler Vertextung, die in der Tat mehrere Lesarten von Scbuld erlaubt, zu tun haben, besteht in der französischen Fassung eine Tendenz zur Aufhebung der Polysemie und damit zur Verengung eines relativ offenen Sinngefüges. Erst in der Ubersetzung des $S$ chloß-Romans verdichtet sich dann der ,Schuld'-Begriff in Richtung auf ein religiöses, metaphysisches oder mystisches Erlebnis im Sinne der erwähnten Vorworte und Kommentare. Es bedarf nicht erst der Anstrengung des Konnotierens, um bei der Schuldfrage auf das Thema der Sünde, ja der Erbsünde zu stoßen, wie das folgende Beispiel

26 Die folgenden Thesen aus einer ïbersetzungstheoretischen Diskussion in den Weimarer Beiträgen 19 (1973), 36, mögen dies verdeutlichen: "Jede Nachdichtung is in nachschaffender und schöpferischer Prozeß [...], eine Interpretation des Originals entsprechend einem bestimmten Treueideal. “ Mit der Ideologisierung des Treueideals" geht der individuelle Impetus der Übersetzerintention freilich zum "Tröen Teil verloren (ebd. 37): Hier wird wirklich neue internationalistische, großten Tell verloren (ebd. 37): "nier wird wirk priit und wird auch eine neue Traditionsbeziehung geschaffen." zeigt (Sch. 179): Du wirst bineingezogen werden in unsere Dinge, unschuldig, nicht viel schuldiger als Barnabas (681): Tu vas te trouver mélé à nos affaires, innocemment, sans plus de péché que Barnabé.

Daß die Interpretation von Schuld als Sünde aufgrund der besonderen Appellstruktur des Textes möglich und wahrscheinlich ist, soll hier nicht in Abrede gestellt werden. Denn daß es sich einerseits um eine allgemeine Schuld handelt, signalisiert das kollektive unsere Dinge, daß andererseits der Held an dieser Schuld sozusagen keine Schuld har, geht aus dem passiven wirst bineingezogen und der für Kafka so typischen Verbindung von affirmativem unschuldig und einschränkendem nicht viel schuldiger hervor. Die Übersetzung entbindet den Leser jedoch zumindest teilweise von dieser analytischen Anstrengung. Wie vorher schon in den satzinternen Verbindungen von culpabilité und délit, culpabilité und crime oder culpabilité und faute wird nun in der Verbindung von coupable und péché das wiederholende Glied durch einen anderen Ausdruck ersetzt. Damit präsentiert sich uns ein Fall von syntagmatischer Substitution, der - besonders weil von der Abweichung ein Leitmotiv betroffen ist - Ausdruck eines bestimmten Interpretationsstandpunktes ist. Bestätigt finden wir das durch ein anderes Beispiel, und zwar ebenfalls aus dem langen Gespräch zwischen Olga und K., das für religiöse Deutungen freilich besonders empfänglich zu sein scheint. Im Original sagt Olga ( $S c h .212)$ : Und bier mag ich eine Schuld in deinem Sinne baben. In der Ubersetzung sagt sie (718): Sur ce point, je peux avoir péché de ton point de vue. Eine genauere Kontextanalyse, die hier nicht nachgeholt werden soll, würde wahrscheinlich ergeben, daß der Ubersetzer in seinem Verständnis von Schuld als Sünde bestimmten Anspielungen des Textes, die er subjektiv auslegt, gefolgt ist, im wahrsten Sinne des Wortes, Rück-sichten' genommen hat.

Ich meine, daß die Ubersetzung ihre Leser in religiös-metaphysischen Deutungen zumindest bestärkt, zumal auch der kritische Apparat der französischen Kafka-Ausgaben - die Analysen von David inbegriffen - die religiöse Komponente entschieden betonen. Wenn Hans Joachim Schoeps, an den theologischen Spürsinn des Lesers appellierend, die These vertritt ${ }^{27}$, die beiden großen Romane Kafkas seien „im Grunde in einer modernen Hieroglyphenschrif geschrieben", die es zu entziffern gelte, so fühlt man sich in diesem Zusammenhang an die symbolische Großschreibung bestimmter Schlüsselwörter in den Ubersetzungen erinnert. Begriffe wie la Loi, l'Ecriture, le Cbâteau werden durch ihre Majuskeln ganz selbstverständlich der Aura eines Mysterium tremendum zugeordnet. Auch auf dieser graphematischen Ebene äußert sich eine durchaus meinungsreflektierende und meinungsbildende Übersetzungsvarianz. Die Großschreibung - sie wird in Davids Ausgabe leider nicht korregiert will hier verstanden sein als Ausdrudk der Distanz, die zwischen Josef $K$. und dem Gesetz oder den Dorfbewohnern und dem Schloß herrscht. Dies sind Deutungsanweisungen an den Leser, die im Original zumindest in dieser Direktheit nicht erscheinen. Bezeichnend ist, daß selbst solche Details sich in

27 Theologische Motive in der Dichtung Franz Kafkas, in: Die newe Rundschau 62 (1951), 32. 
der französischen Kafka-Kritik als religiöse Mystifizierungen oder Mythisierungen direkt niederschlagen, derart, daß die Großschreibung z.B. bei Vialatte ("la Vie", "la Justice", "la Grâce") oder auch bei David ("la Loi", "le (hâteau") in die Kommentare übergeht. Im Gegensatz dazu steht die demonstrative Kleinschreibung von Gilles Deleuze und Felix Guattari ${ }^{28}$. Vor dem Hintergrund der Obersetzungen ist sie freilich ihrerseits eine Tendenzanzeige, wenn nicht gar ein Politikum im eigentlichen Sinne des Wortes. „La loi", kleingeschrieben, bedeutet: Das Gesetz wird vom Himmel zur Erde heruntergeholt und dadurch auch mit kulturellen, sozialen oder schlechthin politischen Kontexten verknüpfbar. Daß das $S c b l o \beta$, neben der TürhüterLegende im Proze $\beta$, am ehesten zu metaphysischen Spekulationen Anlaß gibt, ist offensichtlich. Insbesondere hier wurde das praktiziert, was ich als Verengung des offenen Sinngefüges bezeichnet habe. Dem Offenbarungswort entspricht le texte d'un Evangile (678); die Oberschrift des 15. Kapitels, Bittgänge, ist übersetzt mit Pèlerinages, Sollicitations und - von David in Klammer gesetzt - Implorations (706). Die sinnlosen Bittgänge zum Vorsteher (202) werden zu ses inutiles pèlerinages chez le maire (707). Den Satz Er plagte sich bald auf seinen Gängen (203) übersetzt Vialatte durch Il se tuait en pèlerinages (708). Der Inhalt ist in diesem Kapitelzusammenhang, daß die Aufträge zur Reparatur der Schuhe ausbleiben und nun die Schusterfamilie geächtet wird, nachdem Amalia den in gemeinsten Ausdrücken gehaltenen Aufforderungsbrief von Sortini zerrissen hatte. Die Bittgänge des Vaters waren also zunächst einmal der Versuch einer direkten Kontaktaufnahme mit den Herren vom Schloß, damit man ihm verzeihe und er die für seine Familie lebensnotwendige Arbeit wieder ausführen und die damit verbundene (auch soziale) Integration in die Dorfgesellschaft erreichen könne. Die gleichnishafte Auslegung des Kapitels vollzieht sich hier offenbar bereits auf der Ebene der Ubersetzung.

Freilich soll nicht bestritten werden, daß Kafkas Texte auch voller religiöser Anspielungen stecken. Doch treten solche Anspielungen sehr selten ohne bestimmte Formen der ,Zurücknahme' auf. Ein Mittel der Zurücknahme ist die Ironie. Darauf hinzuweisen, scheint gerade unter übersetzungsspezifischem Aspekt notwendig, wie das folgende Paradigma - es handelt sich um ein Gespräch zwischen K. und dem Wirt im 3. Kapirel - deutlich macht (Sch. 113): „Nein, nun muß aber desbalb nicht gleich Schwefel vom Himmel regnen." „Nein", sagte K., „danach siebt das Wetter nicht aus." Vialattes Übersetzung lautet (611): „Allons, allons, ce n’est pas pour cela que la fin du monde va arriver." - „Non", dit $K$., „le temps qu'il fait n'en laisse rien prévoir." Während bei Kafka im Wörtlichnehmen des Bildes eine ironische Distanz zur Unheil prophezeienden Assoziation gewahrt bleibt ${ }^{29}$, versucht Vialatte unter Verlust

28 Kafka - Pour une litt. mineure, Paris 1975

29 Vol im 6. Kap. das Gespräch zwischen $K$. und der Wirtin, wo sich eine ganz Vgl. im 6. Kap. das Gespräch zwischen K. und der Wirtin, wo sich eine ganz
ähnlich konstruierte Stelle befindet. Der Ausdruck Der Segen war über ibnen, aber man verstand nicht, ibn berunterzubolen wird durch den räumlichen Bezug - die Wirtin lag ausgestreckt auf dem Rücken und blickte zur Decke empor (Sch. 83) ironisiert. der Bildlichkeit und damit der Ironie, die Aussage eindeutig zu gestalten. Dadurch, daß das ,Ende der Welt beim Namen genannt und die ironische Distanz kaum noch aktualisiert wird, erscheint das religiöse Motiv um vieles verpflichtender. Interessant ist diese Stelle auch wegen der syntagmatischen Anknüpfung, denn die Aussage über das Wetter wird zu Beginn des 10. Kapitels wieder aufgenommen (Sch.114): Auf die wild umwehte Freitreppe trat $K$. binaus und blickte in die Finsternis. Ein böses, böses Wetter. Das im Zusammenhang mit der Erzählperspektive erwähnte Spiel von Affirmation und Einschränkung, das beim Leser eine permanente Unsicherheit gegenüber Aussagen und Sachverhalten hervorruft, wiederholt sich hier im Bildbereich. Die meteorologischen Verhältnisse zu Beginn des 10. Kapitels (Finsternis, böses, böses Wetter) scheinen die religiöse Konnotation (Schwefelregen' bzw. ,Weltuntergang") einerseits bildlich $\mathrm{zu}$ bestätigen, andererseits sorgt das zwischengeschaltete danach siebt das Wetter nicht aus für Unverbindlichkeit und Ambivalenz ein Phänomen, das die Ubersetzung überspringt.

Etwas Entsprechendes geschieht in einem kurzen Prosastück, das in einer von Vialatte und Jean Carrive gemeinsam herausgegebenen Sammlung von Erzählungen veröffentlicht wurde ${ }^{30}$. Die kurze Skizze hatte Kafka mir Ein Dornbusch betitelt ${ }^{33}$. Jean Carrive macht daraus in seiner Ubersetzung $L e$ Buisson Ardent. Man kann sich vorstellen, welche Konsequenzen derartige Abweichungen für die Rezeption haben beziehungsweise nicht haben können. Eine Deutung, wie sie der tschechische Literaturwissenschaftler Paul Reimann 1957 anbot, wäre unter den Bedingungen des ,brennenden' Dornbuschs - obwohl man der orthodoxen marxistischen Literaturkritik hier mitunter erstaunliche Auslegefähigkeiten einräumen muß - nicht denkbar. Reimann, der nach Ende der ersten Phase der marxistischen Kafka-Rezeption, die sich noch weitgehend im Dekadenz-Vorwurf erschöptte, aktiv wird, glaubt ${ }^{32}$, daß Kafka durch solche Gleichnisse „seine Auffassung der realen gesellschaftlichen Beziehungen auszudrücken suchte". Die Tatsache, daß der Parkwächter eines - was Reimann ausdrücklich betont - „öffentlichen " Parkes dem im Dorngestrüpp Verstrickten nicht zur Hilfe eilt, bringt er mit dem Phänomen der Entfremdung, "der Auflösung und Entmenschlichung aller menschlichen Beziehungen in den Bedingungen des Kapitalismus" in Zusammenhang ${ }^{33}$. Kafka habe diesen $\mathrm{Zu}$ stand kritisieren wollen. Daß sich die französische marxistische Literaturkritik mit ihrer Kafka-Rezeption nach 1945 zunächst so schwer tat - ich erinnere

30 Franz Kafka: La Muraille de Cbine, Paris 1950.

31 In einem Gespräch mit Gustav Janouch in: Dichter über ibre Dichtungen - Franz Kaflea [Anm. 20] 151 f.) taucht der ,brennende Dornbusch wieder auf. Janouch berichtet, der Buchbinder hätte für eine Ausgabe von Erzählungen Kafkas auf dem beckel einen brennenden Dornbusch und darmnter den Namen Franz Kafka (Name
Denten kursiv) in zart gezeichneter Goldprägung angebracht. Kafka hat mit deutlich sichtbarer Verstimmung reagiert: Ich bin kein brennender Dornbusch. Ich bin keine Flamme. Und im Verlaufe des Gesprächs kommt es dann zu einer Offnung des Bildes, in der die religiöse Konnotation, wenn nicht ausgeschlossen, so doch auf ein neutraleres, ontologisches Feld überführt wird: Ich bin kein Licht. Ich babe mich nur in den eigenen Dornen verrannt. Ich bin eine Sackgasse.

32 Die gesellschafiliche Problematik in Kafkas Romanen, in: Weimarer Beiträge 3 (1957), 598.

3s Ebd. 600 . 
nur an die Diskussion mit dem Titel Faut-il brûler Kafkas? in der kommunistischen Zeitschrift Action ${ }^{34}$ —, mag bis zu einem gewissen Grade auch durch die sprachlichen Mystifikationen der Ubersetzung bedingt gewesen sein. Gesellschaftskritische Deutungen, seien sie nun marxistisch oder nicht, konnten bei einer Ubbersetzung, die die Bittgänge zum Gemeindevorsteher oder zu sonstigen behördlichen Instanzen zu Wallfahrten sublimiert, zumindest nicht spontan entstehen.

Die Ubersezzung, selbst Ausdruck einer bestimmten Urteilsstruktur, ist also fähig, Auffassungsweisen der aufnehmenden oder vermittelnden literarischen und kulturellen Systeme zu kanalisieren. Sie kann zu diesen oder jenen konkreten Denkprozessen herausfordern, andere sogar ausschließen. Auf dieser sekundären Rezeptionsstufe wird ihr kulturpolitischer Aussagewert besonders manifest - ein Problem des wechselseitigen Bezugs von Ubersetzungsanalyse und Rezeptionsforschung, das unter anderem Ulrich Weisstein ${ }^{35}$ und Dionýz Durišin ${ }^{38}$ genauer analysiert haben. Laut Durišin „gehört die Ubersetzung als eine sehr wichtige Äußerung einer interliterarischen Symbiose in die Sphäre der Kontaktbeziehungen" ${ }^{37}$; er fordert "eine konsequente Charakterisierung der ermitrelten Wirkungsformen" ${ }^{88}$. Dazu zählt auch die durch die Obersetzung bewirkte Kritik. Aufschlußreich sind unter diesem Aspekt die ersten französischen Kafka-Rezensionen, weil sich bei ihnen der Kontakt zum übersetzten Werk historisch unmittelbarer gestaltet als bei anderen Rezeptionsformen. In der Besprechung des Schlosses durch Daniel-Rops ${ }^{38}$ finder man zum Teil die in der Ubersetzung festgestellten Akzentuierungen wieder. Die Wirkung des Romans beruht danach auf einer "étonnante tension métaphysique", und die Intention des Autors deutet der Rezensent so $^{40}$ : „atteindre au mystérieux, au secret, à l'ésotérique sans céder au fantastique". Derartige Äußerungen vermischen sich of mit zeittypischem Hintergrundwissen; unter anderem spielt die sich gleichzeitig vollziehende französische Kierkegaard-Rezeption für die Beurteilung des Prager Dichters eine nicht unwesentliche Rolle. Bei DanielRops resultiert daraus ein Dogma, das - neben dem der ,Erbsünde - zu den beständigsten der französischen Kafka-Kritik zählt ${ }^{41}$ : " c'est un univers de l'absurde et qui n'est pensable que tel". Eine metaphysisch-mystische Sehweise herrscht audh bei Claude E. Magny vor, die, was das Schloß betriff, auf die

sa Daniel Biegel: Fawt-il brûler Kafka?, in: Action 99 (26. Juli 1946).

35 Einfübrung in die Vgl. Lit.wiss., Stuttgart 1968. Vgl. das 4. Kap. über „Rezeption“ und Wirkun" 103 ff Weisstein hat diesen Zusammenhang punkruell an einem and "Wirkung", 103 . Weisstein hat diesen Zusamibs Beispiel aus der englischsprachigen Fassung von $A$ mertha deutlich gemacht, wo die Haltung des hochanfgerichtete[n] Vaters (auf der Photographie, die Karl Roßsmann bei sich trägt) mit very erect wiedergegeben wird. Die psychoanalytisch verfahrende Literaturkritik hat aus dieser wörtlichen Ubersetzung die Konsequenz gezogen,
daß Kafka hier die "phallic power" des Vaters habe demonstrieren wollen.

daß Kafka hier die "phallic power" des Vaters habe demonstrieren wollen.
Vgl. Lit.forschung, Berlin-Ost 1976. Vgl. das Kap. Die wichtigsten Typen lit. Beziebungen und Zusammenbänge, 47 ff.

37 Ebd. 65.

38 Ebd. 73.

30 Le Châiear, par Franz Kafka, in: Nowvelle Rev. Franç. 1939, 526-529.

40 Ebd. 526

41 Ebd. 529.
Formel hinausläuft" ${ }^{42}$ " une sorte de mystique sans dieu“. Auf der anderen Seite zeigt sich eine Tendenz zur positiv-religiösen Auslegung der im Werk beobachteten metaphysischen Prozesse. Bemerkenswert scheint, daß selbst Sartre die religiöse Argumentation so eindeutig vertritt ${ }^{43}$ : „Et sans doute, Kafka veut atteindre ici la transcendance du divin, c'est pour le divin que l'acte humain se constitue en vérité." Sartre befindet sich hier in ,Rezeptionsgemeinschaft" sowohl mit Brod als auch mit den Übersetzern, die, wie zum Beispiel Klossowski" "toute ,sécularisation" de Kafka" von sich weisen. Auch für die anhaltende kritische Auseinandersetzung mit dem Problem des, péché originel mögen (neben Brod) die Ubersetzer mitverantwortlich sein. Wie sehr gerade dieses Thema der ,Sünde, das in den Romanen Kafkas (als Begriff) mehrfach erst durch die Ubersetzung eingeführt wird, im kritischen Bewußtsein verankert ist, deutet eine Besprechung der Neuauflage der Vialatte-Übersetzung $\mathrm{an}^{45}$. Zwar macht man, im Jahre 1977, Konzessionen an ein politisches KafkaBild ("le politique et le métaphysique se recoupent"), doch stehen solche Kompromisse im Widerspruch zu jener dezidiert religiösen Lesart, die der Rezensent der französischen Offentlichkeit - gewissermaßen als Affirmation der Obersetzung - schließlich anbietet ${ }^{46}$ : "La notion de péché se révèle un moyen de combattre l'angoisse d'exister et de mourir sans raison."

Diese Thesen der Kritik - wir müssen uns auf wenige Stichproben beschränken - stehen hier nicht als solche zu Diskussion. Sie können indes die spezifische Wirkungsqualität der französischen Kafka-Fassung zumindest andeutungsweise illustrieren. Der Verlauf der kritischen Auseinandersetzung mit dem Dichter, das Kafka-Bild in Frankreich, ist mit dem Ubersetzungsprozeß eng verzahnt - eine Einsicht, die der neueren französischen Kafka-Forschung durchaus nicht fremd ist ${ }^{47}$ : „La plupart des essais et articles français ont souffert d'un certain nombre de maladies pour ainsi dire, congénitales' Écrits par des auteurs qui ont lu Kafka en traduction, ils ne se posent, et pour cause, aucune question sur la langue, alors que chez $\mathrm{Kafka}$ ce problème revêt d'une grande importance; correlativement, ils sont obligés de se fier à des traductions qui, dans le meilleur des cas, (et compte tenu d'exception signalée plus haut [gemeint sind $u$. a. die Ubersetzungen von Marthe Robert]) - se situent dans la tradition des ,belles infidèles."

Die oben beschriebenen Verstehensweisen - und damit möchte ich abschließend einen weiteren Aspekt der Aufnahme Kafkas in Frankreich kurz skizzieren - lassen sich bis zu einem gewissen Grade durch die sogenannte ,Exterritorialitätr des Prager Dichters erklären. Der Mangel an historischer Bestimmtheit, zunächst im Werke selbst, dann aber auch die nur unzulängliche Kenntnis des sozio-kulturellen Kontextes, der biographischen Elemente, ja der

42 Kafka on l'écriture objective de l'absurde - Procès en canonisation, in: Cabiers du Sud, Nov. 1942, 32.

4'Etre et le Néant, Paris 1943, 324.

44 N. P.: Avec le traductetur de Kafka (Entretien avec P. Klossowski), in: Les Lettres Franç. 27, An. 4 und 5, Paris, Oct. 1945.

45 Bertrand Poirot-Delpech: Kafka dans la "Pléiade ${ }^{\alpha}$ in: Le Monde, Sél. hebd. (30.12.76 bis 7.1.77), 12 .

46 Ebd.

47 Claude Prévot: A la rechercbe de Kafka, in: Europe 49 (1971), $13 \mathrm{f}$. 
Sprachzugehörigkeit des Dichters haben sich auf die Aufnahme und Vermittlung seiner Werke sicherlich nicht günstig ausgewirkt. Kafka, so sagt sein Übersetzer Vialatte ${ }^{48}$, „débarquait dans la littérature comme un Martien sur le globe terrestre." Derartige Aussagen sind symptomatisch für ein bestimmtes Vorverständnis. Doch mit einem ins Mystische verschobenen Total-Exil hat Kafkas Prager Situation, wie man - heute - meint, wenig zu tun. Seine Exterritorialität war keine, die außerhalb jedes geschichtlichen Bezuges gelegen und ihn daher durchlässig gemacht hätte für alle möglichen geistigen Annexionsformen bis hin zur "Naturalisierung“ (Marthe Robert). Kafkas ,Purismus', auf den sich manche Übersetzer mit metaphysischem Eifer stürzten, war im Grunde die verdeckte und deshalb unerkannte Geschichtlichkeit seiner Sprache und Kunst. Der angebliche ,Indifferentismus' des Ausdrucks war zum einen die individuelle Reaktion Kafkas auf die Situation in Prag, wo Juden, Tschechen und Deutsche alles andere als eine homogene Sprachgemeinschaft bildeten. Zum anderen reagierte er auf das sprachliche Chaos in den Dichtungen seiner Prager Zeitgenossen. Bei Kafkas Spracharmut handelte es sich vielmehr um Sprachverteidigung ${ }^{49}$. Eine Obersetzung, die freiwillig-schöpferisch oder aus Gründen nationalsprachlicher Interferenzen semantische Offenheit in Bestimmtheit verkehrt, Kafkas Elliosen auflöst, Redundanzen durch neue Begriffe ersetzt und ähnliches mehr - Phänomene, die wir beobachten konnten - erscheint vor diesem Hintergrund besonders problematisch.

Schließlich stellt sich die Frage nach der UUbersetzbarkeit Kafkas überhaupt. Die Stringenz und die, Reinheit ${ }^{c}$ seiner Sprache war vielen Ubersetzern, vor allem Vialatte, Anlaß zu quasi sprach-mystischen UUberlegungen. Kafkas Sprache, meint Vialatte, gehöre einer "vierten Dimension des Universums" an. Der Gedanke an eine Ursprache, an eine Sprache jenseits von sozialer, kultureller oder persönlicher Färbung ist also präsent. Das erinnert an die Konzeption Karl Vosslers, der hinter der Verschiedenheit des Sprechens ein Nichtmehr-Linguales, ein Übersoziales, ja sogar einen Bereich voraussetzt, wo der Ubersetzer "mit der metaphysischen Sprachgemeinschaft der Menschheit und

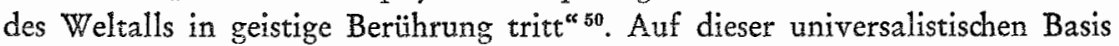
ist jede Ubersetzung legitim. Entsprechend verbirgt sich hinter den Worten von Vialatte, wonach Kafka „Europa eine neue Sprache" geschenkt habe, der Glaube an eine die Sprechgrenzen, die kulturellen oder sozialen Grenzen überschreitende Sprachpotenz des Dichters. Die Gegenposition ist die monadische, die von dem Manifesten, dem kulturell, sozial usw. jeweils Fixierten in der Sprache ausgeht und im Extremfall daraus das Gesetz der Unübersetzbarkeit

48 Introduction, aaO. [Anm. 24] 32.

40 Die These vom defensiven Charakter der Sprache Kafkas bezieht sich auch auf die Entwicklung im eigenen Werk, das in den Anfängen, vor allem in Beschreibung eines Kampfes [ $=B$.$] von ungebräuchlichen Wendungen und von Pragismen nicht$ frei ist. Daraus entstehen besondere übersetzerische Probleme: Unübersetzbar sind die Erweiterungen des Reflexivums (Da er sich rïlpste $[B .40]$ ), der weggelassene Artikel (Kleider [...], die über schönen Körpern schön sich legen [B. 40], bestimmte lexilkalische Eigenarten (Ich gab die Hände von meinen Obren [B, 28]) und ähnliches mehr.

so Sprachgemeinschaft als Gesinnungsgemeinschaft, in: Das Problem des Übersetzens, hg. v. H. J. Störig, Darmstadt 1963, 203. ableitet. Eine solche Haltung vertritt offenbar Klaus Wagenbach in bezug auf Kafka, wenn er betont ${ }^{51}$, daß Kafka "unübersetzbar ${ }^{\text {" }}$ und daher die Wirkung des Prager Dichters auf Grund von Übersetzungen "höchst fragwürdig " sei.

Die Ubersetzungsproblematik läßt sich freilich nicht nur von diesem soziokulturellen Aspekt her, der auf die vielfach postulierte Unbestimmtheit Kafkas ein etwas anderes Licht wirf, erfassen. Problematisch ist jede Kafka-Übersetzung wegen der im T'ext angelegten semantischen Offenheit. Hier hat der Autor in der Tat wenig Rücksicht auf seinen Ubersetzer genommen. Dieser befindet sich als Interpret in der Situation jener Person, die in der Parabel den Schutzmann nach dem Weg fragt und doch nur sich selbst überlassen bleibt. Wir haben andeutungsweise gesehen, welche Konsequenzen dies für die kommunikativen Prozesse hat, die zwischen der Produktion des Originals und der Rezeption der Ubersetzung abrollen.

51 Franz Kafka, Bern 1958, 89. 